\title{
Interstitial MRI lymphangiography of the lower
}

\section{limbs}

Dear Sir,

We read the article "Technical note: MRI lymphangiography of the lower limb in secondary lymphedema" by Kamble et al., published recently in your journal with great interest. ${ }^{[1]}$ We concur with the authors that the investigation of secondary lymphedema has been enigmatic with direct lymphangiography being too invasive and lymphiscintigraphy having poor spatial and temporal resolution. The authors have rightly pointed out that indirect contrast-enhanced MRI lymphangiography offers excellent visualization of the lower limb lymphatics in secondary lymphedema. The greatest advantage of this technique is the absence of radiation exposure, the multiplanar capability of MRI allowing for precise compartmental localization (epifascial/subfascial/intramuscular) and accurate depiction of the extent of abnormality. We would like to point out that one of the major limitations of the technique is poor visualization of inguinal lymph nodes after intradermal injection of contrast in the feet. We would also like to

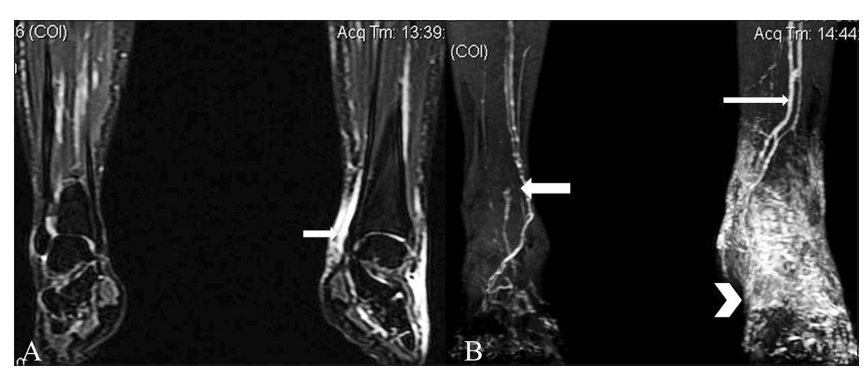

Figure $1(A, B)$ : Coronal short tau inversion recovery (STIR) image (A) shows extensive epifascial edema in the left foot of a 35-year-old female patient with secondary lymphedema (thick arrow). Coronal 3D maximum intensity projection (MIP) reconstruction image of a 3D time-of-flight (TOF) acquisition, 15 min after intradermal gadobenate dimeglumine contrast injection shows paucity of axial lymphatics in the foot and lower leg region with enlargement of the remaining channels (thin arrow) and extensive dermal back flow in the foot region (arrowhead). The right foot shows normal caliber lymphatic channels (thick arrow in B). share our experience of indirect MRI lymphangiography with a newer MRI contrast agent with high relaxivity, gadobenate dimeglumine $(0.5 \mathrm{mmol} / \mathrm{L}$ MultiHance, Bracco, Milano, Italy). We have found gadobenate dimeglumine to provide more detailed depiction of the lymphatic anatomy in the small number of patients that we have done [Figure 1A and B]. Lohrmann et al. have demonstrated frequent visualization of inguinal lymph nodes after injection of gadoteridol (ProHance, Bracco-Altana, Konstanz, Germany) one of the newer contrast agents. ${ }^{[2]}$ The use of newer contrast agents with higher relaxivity thus offers the possibility of simultaneous depiction of the lymphatic channels and the lymph node groups.

\section{Anit Parihar, Swastika Suvirya', Suresh Kumar², \\ Ragini Singh}

Departments of Radiodiagnosis and Imaging, 'Dermatology, Venereology and Leprosy, and ${ }^{2}$ General Surgery, Chhatrapati Shahuji Maharaj Medical University, Chowk Lucknow,

Uttar Pradesh - 226003 , India E-mail: anitparihar@gmail.com

\section{References}

1. Kamble RB, Shetty R, Diwakar N, Madhusudan G. Technical note: MR Lymphangiography of the lower limb in secondary lymphedema. Indian J Radiol Imaging 2011;21:15-7.

2. Lohrmann C, Foeldi E, Bartholomae JP, Langer M. Gadoteridol for MR imaging of lymphatic vessels in lymphoedematous patients: Initial experience after intracutaneous injection. Br J Radiol 2007;80:569-73.

\begin{tabular}{|l|l|}
\hline \multicolumn{2}{|c|}{ Access this article online } \\
\hline Quick Response Code: & \\
\hline & Website: \\
\hline & www.iji.org \\
\cline { 2 - 3 } & DOI: \\
\hline
\end{tabular}

